Check for updates

Cite this: RSC Adv., 2017, 7, 54246

Received 3rd August 2017

Accepted 19th November 2017

DOI: $10.1039 / \mathrm{c} 7 \mathrm{ra0} 8595 \mathrm{~h}$

rsc.li/rsc-advances

\title{
Electrospun nanofibrils embedded hydrogel composites for cell cultivation in a biomimetic environment $\dagger$
}

\author{
Sol Lee, ${ }^{a}$ Hye Sung Kim ${ }^{b}$ and Hyuk Sang Yoo (iD *ab
}

Hybrid composites composed of nanofibrils and hydrogels were fabricated for cell cultivation in a cellfriendly environment mimicking the extracellular matrix. Electrospun fibrous mats composed of polycaprolactone (PCL) were digested to nanofibrils (NF) under optimized conditions and subsequently incorporated into hydrogels composed of gelatin and alginate. The hydrogel hybrids containing nanofibrils (NF/hydrogel) were calcium-crosslinked and solidified with or without cells. The mechanical properties of the cell-incorporated NF/hydrogels were dramatically increased in proportion to the NF content in the NF/hydrogels after 7 days of cultivation. Mass erosion of the NF/hydrogel was significantly attenuated when NF content increased. Confocal microscopy revealed that fibroblasts cultivated in NF/ hydrogels showed superior adhesion behavior onto the matrix compared to those without NF, and the formation of spindle and stress fibers was distinct in NF/hydrogels with a high NF content. Cells on NF/ hydrogels showed higher levels of collagen synthesis as NF content increased. Thus, NF/hydrogels are expected to serve as a cell culture matrix facilitating cell-matrix interactions by combining supportive nanofibrils and hydrogels.

\section{Introduction}

Extracellular matrix (ECM) mimetic structures have received much attention as fabricators of tissue-cultivating matrices for 3-D cell cultures with aims to maintain and enhance tissuespecific phenotypes of the respective cell types. ${ }^{1,2}$ Among the biomimetic structures of the ECM, fibrous structures have been extensively explored because of their structural similarity to natural ECM structures such as the collagen, elastin, fibronectin, and proteoglycan constituting the basal lamina. Since these fibrous ECMs provide mechanical support as well as differentiation niches, the relationship between the ability of these molecules and biomimetic materials to proliferate and differentiate cells in "home-like" environments for extended periods of time has been extensively studied., ${ }^{2,3}$ Various proteins and carbohydrates constituting the ECM were extensively coated and mixed with biomaterials to fool the body's systems into recognizing the manipulated biomaterials as natural ECMs. Reprogrammed stem cells cultivated on collagen IVcoated materials were differentiated into cardiovascular and hematopoietic lineages. In another study, a mixture of collagen ${ }^{a}$ Department of Biomedical Materials Engineering, Kangwon National University,
Chuncheon, 24341, Republic of Korea. E-mail: hsyoo@kangwon.ac.kr
${ }^{b}$ Institute of Bioscience and Biotechnology, Kangwon National University, Republic of
Korea

$\dagger$ Electronic supplementary information (ESI) available. See DOI: 10.1039/c7ra08595h
I and chondroitin sulfate on polymeric scaffolds was proven to regenerate new calvarial bone. Many approaches have been proven to be successful, however, most approaches have innate issues associated with them, such as immune response and the high expense of producing such bio-originated materials. ${ }^{2}$

Due to structural similarity to the natural ECM, electrospun nanofibers were extensively tailored to cultivate, proliferate, and differentiate various cells in biomimetic environments..$^{6-8}$ Thus, polymeric meshes composed of the nanofibers with a diameter of several hundred nanometers have been cultivated with stem cells and primary cells and decorated with many biological cues. Osteogenic differentiation was accomplished with nanofibers composed of PCL and chitosan for ECM-resembling cultivation. ${ }^{4}$ Cell adhesion and osteogenic markers were more prominently observed in cells on nanofibers than on flat surfaces. We previously fabricated nerve growth factorimmobilized nanofibers to cultivate and differentiate mesenchymal stem cells. ${ }^{5}$ After 5 days cultivation, stem cells on aligned nanofibers were differentiated to nerve-like cells with an elongated shape compared to those on randomly distributed fibrous structures. In another study, a pheochromocytoma cell line (PC12) was cultivated on aligned electrospun nanofibers coated with protein and was finally differentiated to nerve cells on the nanofibers. ${ }^{6}$ A nanofibrous conduit was fabricated for axon regeneration in spinal cord injuries using aligned nanofibers. ${ }^{7}$ Aligned nanofibers embedded in collagen scaffolds enhanced myelination and axonal regeneration at injury sites of spinal cord. In another study, fibroblasts were cultivated in 
electrospun nanofibers to obtain ECM-coated scaffolds after decellularization. ${ }^{8}$ Mature cardiomyocytes were differentiated by the decellularized ECM from fibroblast cultivation. These studies accordingly indicate that cell proliferation and fates are largely dependent on the topographical cues of nanofibers as well as the immobilized ligands. However, most matrices composed of nanofibrous meshes do not allow cells to infiltrate and migrate inside the matrix because inter-fibril spaces are not large enough. Although the high surface area-to-volume ratios of nanofibers are considered to effectively facilitate matrix-cell interactions, cell-to-cell interactions in 3-D environments are not easily formed because cells are not allowed inside the matrix.

Physical hydrogels provide water-rich environments and mechanical support to the encapsulated cell within the matrix, and thus can be ideal materials for tissue engineering. However, the mechanical strength of physical hydrogels is weaker than that of covalently-tethered hydrogels, although physical hydrogels have superior biodegradability. To overcome the mechanical disadvantages of physical hydrogels, several attempts have been made to increase chain entanglements of hydrogels by improving hydrophobic interactions and electrostatic interactions. ${ }^{9-11}$ Nanofibers were also introduced to hydrogels, forming hybrids composed of nanofibers and hydrogels. ${ }^{10}$ These nanofiber lattices increased the tensile strength of the composite scaffolds. We previously electrospun polycaprolactone nanofibers to form nanofibril-cell composites for observing the celldirected association of nanofibrils. ${ }^{\mathbf{1 1}}$ Electrospun nanofibers were successfully hydrolyzed to nanofibrils in optimized conditions and surface-decorated with poly(methacrylate) derivatives for sequestrating serum proteins. These fibrils spontaneously associated with cultivating cells to form cellmatrix clusters.

In the current study, we fabricated a hybrid matrix composed of nanofibrils and hydrogels to provide contact points and differentiation cues to cells cultivated in 3-D environments. This hybrid matrix allowed cells to recognize both fibril structures and water-rich environment that simulate cells in the ECM. We measured the mechanical properties and mass erosion of the cell matrix to compare the effects of nanofibrils on cell matrix assembly. Morphological differences in cells were also compared to determine the effects of nanofibrils on cell attachments and stretching. Finally, we evaluated the gene expression profiles of cells to provide evidence for cell matrix assembly.

\section{Experimental}

Materials

Polycaprolactone (PCL, $M_{\mathrm{w}}=43 \mathrm{kDa}$ ) was purchased from Polysciences (Warrington, PA). An alginic acid sodium salt (low viscosity) was purchased from Alfa Aesar (Haverhill, MA). Gelatin from porcine skin (Type A) was purchased from Sigma Aldrich (St. Louis, MO). Calcium chloride anhydrous $\left(\mathrm{CaCl}_{2}\right)$, methanol, chloroform, and ethanol were purchased from Daejung Chemicals and Materials (Siheung, Republic of Korea). Dulbecco's modified Eagle's medium (DMEM), fetal bovine serum (FBS), and Dulbecco's phosphate buffered saline (PBS) were purchased from Gibco BRL (Staley Rd Grand island, New York). Murine fibroblast cell line NIH3T3 cells was purchased from the Korean Cell Line Bank (Seoul, South Korea.). Thiazolyl blue tetrazolium bromide (MTT) was purchased from Daeil Biotech. (Solon, OH). Hipi Plus $5 \times$ PCR Premix containing Taq and Reverse Transcription (RT) Premix $(5 \times, 20 \mu \mathrm{L}$ reaction $\mathrm{M}$ MLV-RT, RNase $\mathrm{H}+$ ) were purchased from Elpis Biotech (Daejeon, Korea).

\section{Method}

Preparation of PCL nanofibrils. PCL nanofibrils were prepared by hydrolyzing electrospun nanofibrous meshes in a sodium hydroxide $(\mathrm{NaOH})$ solution of an optimized concentration. ${ }^{11}$ PCL (2.5) dissolved in a chloroform/methanol $(10 \mathrm{~mL}$, $3: 1(\mathrm{v} / \mathrm{v}))$ mixture was loaded into a $10 \mathrm{~mL}$ syringe and ejected through a blunt needle $(25 \mathrm{G})$ at a flow rate of $1 \mathrm{~mL} \mathrm{~h}^{-1}$. The applied voltage between the needle and the grounded aluminum foil was $+15 \mathrm{kV}$ at a $15 \mathrm{~cm}$ distance. Nanofibers on foil were detached with ethanol and then milled for $30 \mathrm{~s} 3$ times. The milled nanofibers were hydrolyzed in a sodium hydroxide solution ( $1 \mathrm{M}$ ) for $6 \mathrm{~h}$ and the obtained nanofibrils were washed with distilled water 3 times to remove salts. The length of the nanofibers was monitored by optical microscope every $1 \mathrm{~h}$ during the hydrolysis process.

Preparation of nanofibril-fortified hydrogels and scanning electron microscopy (SEM). Nanofibril-fortified hydrogels were prepared by calcium crosslinking a mixture composed of alginate, gelatin, and PCL nanofibrils as previously described in the literature with minor modifications (Scheme 1). Briefly, alginate $(0.268 \mathrm{~g})$ dissolved in serum-free DMEM $(5 \mathrm{~mL})$ at room temperature was mixed with a gelatin solution, which was prepared by dissolving gelatin $(0.330 \mathrm{~g})$ in the same solvent (5 mL) at a volume ratio of $2: 1$ at $40{ }^{\circ} \mathrm{C}$ while stirring, and then cooled down to $37^{\circ} \mathrm{C}$. The nanofibrils were sterilized by inversion in $70 \%$ ethanol overnight and washed with PBS 3 times during centrifugation (13000 rpm, $20 \mathrm{~min}$ ), and were then added to the alginate/gelation solutions at $10-200 \mathrm{mg} \mathrm{mL}^{-1}$ (nanofibrils/hydrogel, w/v) and uniformly mixed by vortexing (Table 1). The mixture was transferred to a 96-well plate and crosslinked by $0.5 \% \mathrm{CaCl}_{2}$ solution for $3 \mathrm{~h}$. To prepare cellencapsulated hydrogels, NIH3T3 $\left(25 \mu \mathrm{L}, 1 \times 10^{4}\right.$ cells $)$ in DMEM were added to fibril-Alg-Gel solution $(75 \mu \mathrm{L})$ and crosslinked in hydrogels by $0.5 \%$ calcium chloride in a $96-w e l l$ plate. The cell laden hydrogels were washed with $200 \mu \mathrm{L}$ of PBS 2 times and incubated in DMEM $(200 \mu \mathrm{L})$ supplemented with $10 \%$ FBS and penicillin/streptomycin in a $5 \% \mathrm{CO}_{2}$ atmosphere at $37{ }^{\circ} \mathrm{C}$. The media was replaced every two days. The morphology of the hydrogels was confirmed with ultra-high resolution scanning electron scanning microscopy (Hitachi, S4800, Japan) in the Central Laboratory of Kangwon National University (Korea).

Cell proliferation in $\mathbf{N F} / \mathbf{h y d r o g e l s}$. Viabilities of the incorporated cells within NF/hydrogels were determined by an MTTbased cytotoxicity assay. Calcium-crosslinked hydrogels in a 96well plate were washed with $200 \mu \mathrm{L}$ of PBS 2 times and 
A

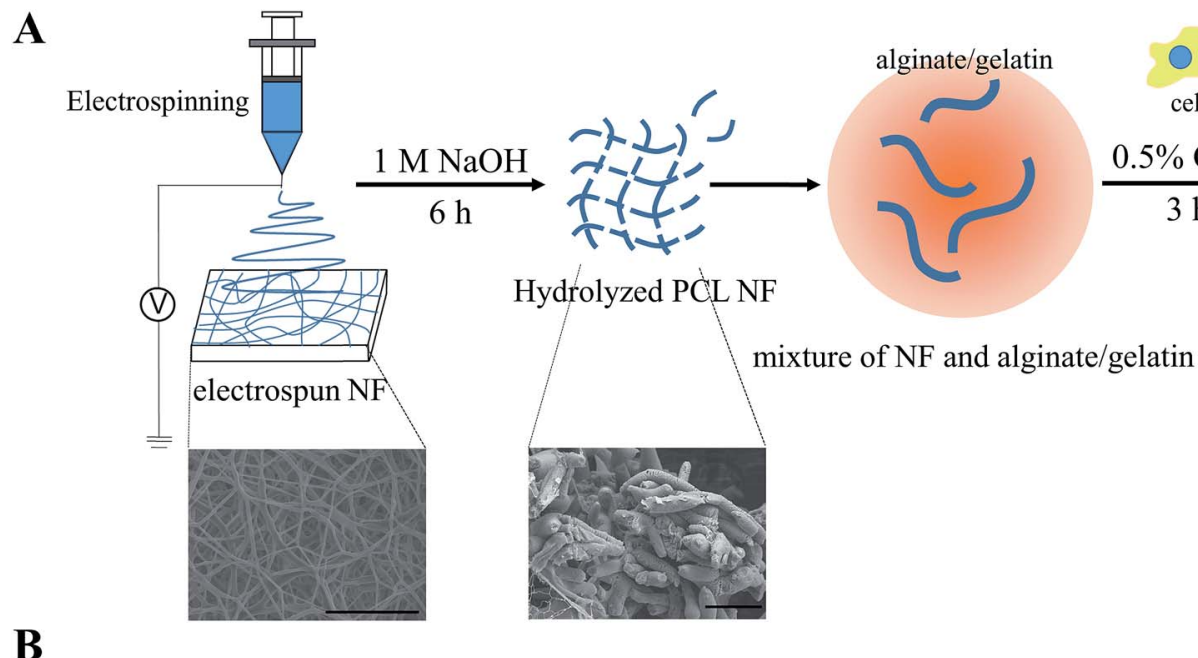

B

Cells in Hydrogel

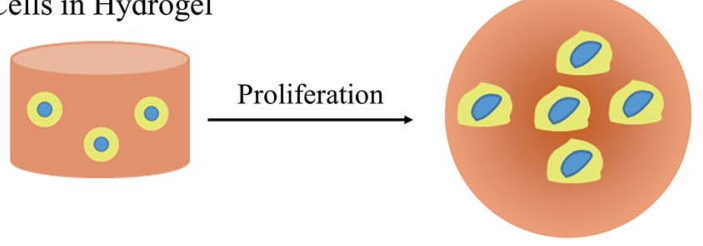

Cells in NF/Hydrogel
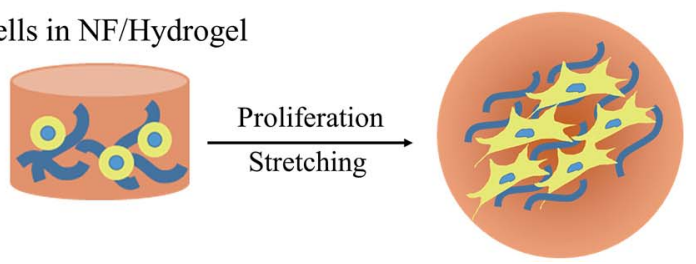

Scheme 1 Schematic presentation of preparing NF/hydrogel encapsulating cells. (A) PCL nanofibers fabricated by electrospinning were milled and hydrolyzed by a high speed grinder and $1 \mathrm{M} \mathrm{NaOH}$, respectively. Hydrolyzed nanofibrils (NF) were homogeneously mixed with hydrogels composed of alginate and gelatin. Cells were finally added to the NF/hydrogel before crosslinking by $\mathrm{CaCl}_{2}$. Inserted images are SEM images of nanofibers and nanofibrils. (B) Anticipated effects of NF/hydrogels on proliferation and stretching of cells in the matrix in comparison to those in hydrogels.

Table 1 Nanofibril-fortified hydrogel (NF/hydrogel) containing various amounts of NF. Compositions of respective hydrogels were presented the weight in $0.1 \mathrm{~mL}$ of the matrix and \#\% NF/hydrogels describe a weight $(\mathrm{w} / \mathrm{v})$ of $\mathrm{NF}$ in the hydrogel

\begin{tabular}{lccc}
\hline NF/hydrogel & $\begin{array}{l}\text { The amounts } \\
\text { of gelatin }(\mathrm{mg})\end{array}$ & $\begin{array}{l}\text { The amounts of } \\
\text { alginate }(\mathrm{mg})\end{array}$ & $\begin{array}{l}\text { The amount of } \\
\text { nanofibrils }(\mathrm{mg})\end{array}$ \\
\hline Hydrogel & \multirow{2}{*}{ (.55 } & 2.68 & 0.00 \\
1\% NF/hydrogel & & & 1.00 \\
$5 \% \mathrm{NF} /$ hydrogel & & & 5.00 \\
10\% NF/hydrogel & & & 10.00 \\
15\% NF/hydrogel & & 15.00 \\
20\% NF/hydrogel & & 20.00
\end{tabular}

incubated in DMEM $(200 \mu \mathrm{L})$ supplemented with $10 \%$ FBS and penicillin/streptomycin in a $5 \% \mathrm{CO}_{2}$ atmosphere at $37^{\circ} \mathrm{C}$. The media was replaced every two days. Cell viability was measured at day 1 , day 3, and day 14 by an MTT assay. After replacing the medium, $10 \mu \mathrm{L}$ of MTT solution $\left(5 \mathrm{mg} \mathrm{mL}^{-1}\right)$ was added to each well and further incubated for $4 \mathrm{~h}$. Formazan crystals were dissolved by $200 \mu \mathrm{L}$ of DMSO and the absorbance was measured at $540 \mathrm{~nm}$ by a plate reader (Thermo Scientific ${ }^{\mathrm{TM}}$ Multiskan $^{\mathrm{TM}}$ GO Microplate Spectrophotometer, Thermo Fisher Scientific) to assess the viabilities. Viabilities were normalized with respect to those of cells cultivated in $\mathrm{NF} /$ hydrogels without nanofibrils at day 1 .

Rheological properties of hydrogels. To determine the effects of nanofibrils on overall mechanical properties of NF/hydrogels, the storage modulus $\left(G^{\prime}\right)$ and loss modulus $\left(G^{\prime \prime}\right)$ of NF/hydrogels were measured before and after cell proliferation $(n=3)$. Two hundred microliters of NF/hydrogels with or without cells were mounted between a cone and a plate and $100 \mathrm{~Pa}$ of compression force was applied at frequency ranges of 0.1 to $10 \mathrm{~Hz}$ in a rotational rheometer (Gemini 200, Bohlin Instruments, Westborough, MA). Each modulus value was expressed as a mean \pm standard deviation.

Mass erosion. Mass erosion of hydrogels was determined by monitoring the change in dry weight of the hydrogels for 14 days. Briefly, hydrogels encapsulating cells $(0.5 \mathrm{~mL})$ in 96 -well plates were incubated in $1 \mathrm{~mL}$ of DMEM and cell culture media was replaced every $24 \mathrm{~h}$. At predetermined intervals $(1,3,7$, and 14 days), each hydrogel was harvested, lyophilized, and weighed. For each group, five devices were employed to measure the weight and the mass erosion was calculated by the following equation:

$$
\text { Mass erosion }(\%)=\left(W_{\mathrm{i}}-W_{\mathrm{a}}\right) / W_{\mathrm{i}} \times 100
$$

where $W_{\mathrm{i}}$ and $W_{\mathrm{a}}$ indicate the initial weight of hydrogels and weight after incubation, respectively.

Confocal laser scanning microscopy (CLSM). To observe the cell morphology in the $\mathrm{NF} /$ hydrogels, cells cultivated in the matrix were microscopically observed by CLSM after $72 \mathrm{~h}$ cultivation. Cells in NF/hydrogels were fixed with a $3.7 \%$ formaldehyde solution in PBS $(0.2 \mathrm{~mL})$ for $40 \mathrm{~min}$ and then washed with PBS three times. After permeabilization in $0.1 \%$ Triton X- 
$100(0.2 \mathrm{~mL})$ in PBS for $2 \mathrm{~min}, \mathrm{~F}$-actin and the nucleus were stained with $5 \mathrm{~mL}$ of Alexa Fluor 568-Phalloidin (300 units per $\mathrm{mL}$ )

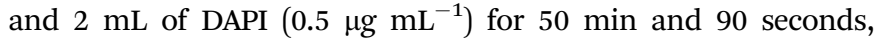
respectively. The stained cells were excited with a HeNe laser (405 $\mathrm{nm}$ ) and an Ar laser (561 nm), and the emission signals were detected with a filter $(411-691 \mathrm{~nm})$ and a filter $(568-691 \mathrm{~nm})$ for DAPI and Alexa Fluor 568-Phalloidin, respectively (LSM 880, Carl Zeiss, Germany). We also evaluated the degree of stretching $\left(D_{\mathrm{S}}\right)$ and the perimeter of individual cells by analyzing the CLSM images (Image J., v1.46, National Institutes of Health, USA). $D_{\mathrm{s}}$ was calculated by dividing the long axis $\left(L_{\mathrm{L}}\right)$ by the short axis $\left(L_{\mathrm{S}}\right)$;

$$
D_{\mathrm{s}}=\left(L_{\mathrm{L}} / L_{\mathrm{S}}\right)
$$

and the perimeters were obtained by measuring the length of the cell membrane of each cell $(n>10)$.

RT-PCR. To compare gene expression levels of cells cultivated in their respective matrices, cells in $\mathrm{NF} /$ hydrogels were incubated for 2 weeks and mRNA was extracted with $1 \mathrm{~mL}$ of TRIzol reagent as described in the manufacturer's protocol. The extracted mRNA was then dissolved with RNase-free water, and the absorbance was measured at $260 \mathrm{~nm}$ (Nanovue, Biochrom, Harvard bioscience, Inc., USA). RT-PCR was used to compare the expression of collagen type I and type II of cells in hydrogels. cDNA was synthesized using $10 \times$ PCR buffer, dNTP mix, primers, and a Taq. PCR solution $(20 \mu \mathrm{L})$ prepared with HIPI $5 \times$ PCR Premix ( $4 \mu \mathrm{L})$ and cDNA (100 ng). The process was run for 40 cycles with an initial denaturation step, a denaturing step, an annealing step, and an extending step. The initial denaturation step was run for $3 \mathrm{~min}$ at $95{ }^{\circ} \mathrm{C}$ and the denaturing step was run at the same temperature for 30 seconds. The extending step was run at $72{ }^{\circ} \mathrm{C}$ for 55 seconds. In the annealing step, each gene (GAPDH, collagen type I, and collagen type II) was run under different temperature conditions for 30 seconds. GAPDH had an annealing temperature of $57^{\circ} \mathrm{C}$, Col I had $62{ }^{\circ} \mathrm{C}$, and Col II had $67^{\circ} \mathrm{C}$. The sense (s) and antisense (as) sequences of collagen type I $\left(5^{\prime} \rightarrow 3^{\prime}\right)$ were CTCCAACGAGATCGAGATC and GTTTACAGGAAGCAGACAGG, respectively. ${ }^{12}$ The sequence of collagen type II $\left(5^{\prime} \rightarrow 3^{\prime}\right)$ were GGGTCTCCTGCCTCCTCCTGCTC $(\mathrm{s})$ and TCCTTTCTGCCCCTTTGGCCCTAATTTTCGGG (as). ${ }^{13}$ The sequences of GAPDH were TCACTGCCACCCAGAAGAC (s) and TGTAGGCCATGAGGTCCAC (as). ${ }^{13}$ The PCR products were analyzed on a $1.2 \%$ agarose gel and the band intensities were quantified with image analysis software for semi-quantitative analysis of Col I and Col II with respect to GAPDH (Image J.).

Statistical analysis. All results are expressed as the mean \pm standard deviation. The statistical significance between a pair of groups was determined by conducting a one-way ANOVA with the SigmaPlot 12.0 software (SPSS); $p<0.05$ was considered statistically significant.

\section{Result and discussion}

Scheme 1 is a schematic presentation of the fabrication of NF/ hydrogels composed of electrospun nanofibrils and gelatin/ alginate hydrogels. Although electrospun nanofibers have been widely used to cultivate many types of cells, multidimensional cultivation of cells in these matrices was considerably limited because of cell-impermeable nanoporous structures. Therefore, in this study, we fragmented electrospun nanofibrous meshes into nanofibrillar structures so that they could be homogeneously distributed along crosslinkable hydrogels. We first hydrolyzed PCL nanofibers under optimized conditions as previously described, and obtained nanofibrils of 8-12 $\mu \mathrm{m}$ in length. ${ }^{11}$ The average length and thickness of the nanofibrils were $12.04 \pm 2.80 \mu \mathrm{m}$ and $2.33 \pm 0.33 \mu \mathrm{m}$, respectively. Because these nanofibrils showed higher waterwettability than electrospun nanofibers, they were homogeneously blended with gelatin/alginate and finally crosslinked to $\mathrm{NF} /$ hydrogels to encapsulate cells for 3-D cultivation. As shown in Table 1, we prepared various $\mathrm{NF}$ /hydrogels with various $\mathrm{NF}$ content levels in the matrix while the amounts of gelatin and alginate was fixed at $5 \%(\mathrm{w} / \mathrm{v})$ and $4 \%(\mathrm{w} / \mathrm{v})$, respectively. We

\section{$\mathrm{NF}$ contents in NF/hydrogel}
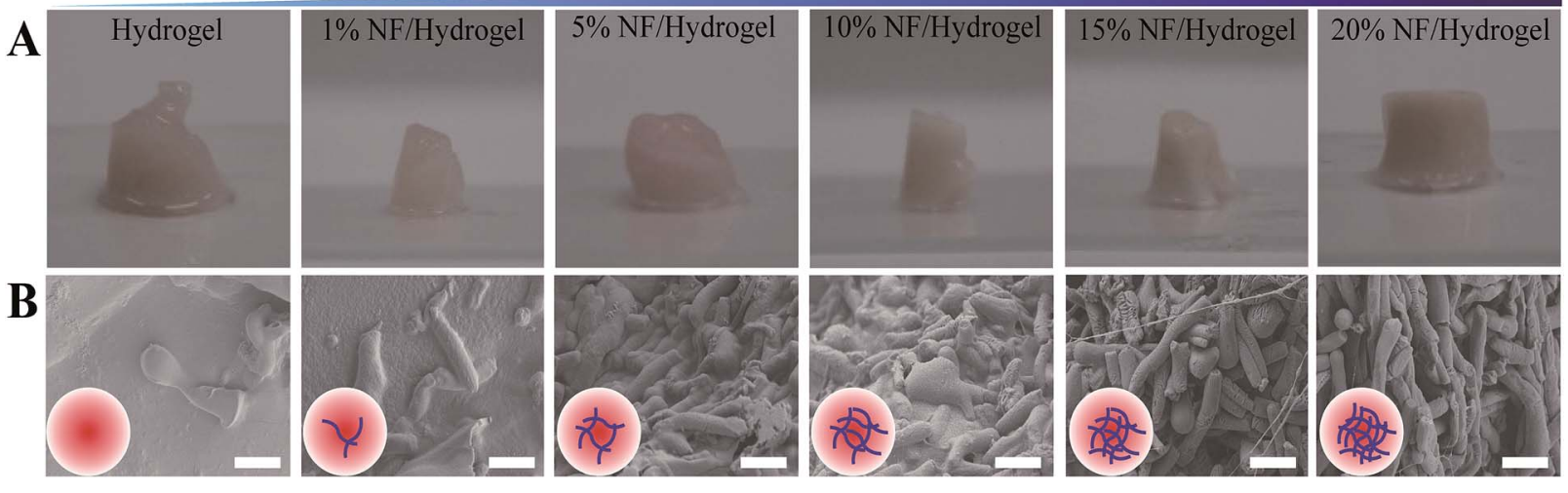

Fig. 1 (A) Morphology of NF/hydrogels. After calcium crosslinking for $3 \mathrm{~h}$, each image was obtained for hydrogel without NFs, 1\% NF/hydrogel, $5 \%$ NF/hydrogel, 10\% NF/hydrogel, 15\% NF/hydrogel and 20\% NF/hydrogel. The volume of NF/hydrogel was maintained at $0.1 \mathrm{~mL}$. (B) Scanning electron microscopy (SEM) of NF/hydrogels with various blend ratios of NF. NF/hydrogels were horizontally cross-sectioned, freeze-dried, and coated with iridium. Insert images are scheme of hydrogel with increasing NF contents. Scale bars are $10 \mu \mathrm{m}$. SEM images at low magnification were also presented to clarify overall morphology of cross-sectioned NF/hydrogels (Fig. S1 $\uparrow$ ). 
confirmed that all $\mathrm{NF} /$ hydrogels were crosslinked and formed physical hydrogels in $0.5 \%$ calcium chloride solution and employed these matrices in further analyses. Fig. 1A shows the shapes of NF/hydrogels with various levels of NF content when the mixtures solidified in 96-well plates were reverted. Although all $\mathrm{NF} /$ hydrogels apparently showed a similar shape, rigidity among the different $\mathrm{NF} /$ hydrogel matrices was different. We saw that the rigidity gradually increased when NF content increased from $0 \%$ to $20 \%$ and the shape of the initial hydrogels showed minor changes accordingly; time-lapse photos are not presented, but Fig. 1A clearly indicates that NF/hydrogels with higher NF content retained the initial shape while those with lower NF content showed irregular shapes. SEM images also confirmed that the NF were homogeneously blended with the surrounding hydrogels according to the blend ratios. NF/ hydrogels showed highly-porous structures and incorporation of NF slightly decreased the pore size (Fig. S1†). Thus, we speculate that these NF can provide mechanical support for the matrix by reinforcing the physical hydrogels. In comparison to alginate and gelatin, PCL NF are more effective for inter-fibrous tethering because of their hydrophobicity; hydrophobic interactions play a significant role in reinforcing the surrounding hydrogel network composed of gelatin/alginate. ${ }^{14}$ Previous studies showed mass erosion of alginate/gelatin hydrogels with artifacts. Artifacts containing hydrogels showed less mass erosion than artifacts not containing hydrogels. Substrates with artifacts created by physical blending or by formation of a semiinterpenetrated network have more mechanical strength.

Fig. 2 shows the mechanical properties and mass erosion of calcium-crosslinked $\mathrm{NF} /$ hydrogels incorporating cells for 1-2 weeks. At day 0 , cell-incorporated $\mathrm{NF} /$ hydrogels showed a decreased elastic modulus when NF content in the matrix was between $0 \%$ and $20 \%$; hydrogels without NF showed the highest elastic modulus values, while $20 \% \mathrm{NF} /$ hydrogels showed the lowest and most unstable modulus at $0.1-20 \mathrm{~Hz}$. However, when cells were cultivated in $\mathrm{NF} /$ hydrogels for 7 days, the profile of the elastic modulus was greatly changed according to the NF content. For hydrogels without NF, the elastic modulus was rapidly attenuated for 7 days and decreased to the lowest level among the matrices. Interestingly, the elastic modulus of $20 \%$ $\mathrm{NF} /$ hydrogels was dramatically increased compared to the modulus at day 0 , suggesting that the cultivating cells greatly enhanced the mechanical properties of NF/hydrogels. Enhancement of the elastic modulus was proportional to the amounts of NF in the NF/hydrogels; in fact, all NF/hydrogels experienced medium- or high-degree enhancements according to NF content. Previously, several studies reported that cell functions were greatly enhanced when anchorage-dependent cells were cultivated on harder surfaces. ${ }^{15-17}$ Cells cultured on stiff matrixes as opposed to softer matrixes exhibited increased expression of many kinds of protein such as actin, tubulin, and pfkp-1. Also, proteins like paxillin and actin exhibited greater expression in cancer cells grown on substrates with higher rigidity than they did on substrates with lower rigidity. Additionally, cells on rigid matrices stretched well. ${ }^{18,19}$ We previously tested the feasibility of PCL nanofibrils as a cell cultivating matrix in 3-D environments. ${ }^{11}$ In our study, we observed that PCL nanofibrils strongly induced self-assembly of the cultivated cells without additional processes within 7 days. Thus, we speculate that the similar tethering behaviors between cells and $\mathrm{NF}$ can cause enhancements to the elastic modulus of NF/ hydrogels. Because integrin-mediated cell-to-cell or cell-tomatrix interactions are responsible for the majority of cell attachment behaviors, integrin molecules on cell surfaces could strongly bind to the cell-secreting ECM and result in tight complexes between ECM-bound NF and cultivated cells. These resultant cell/NF matrices are therefore thought to increase the elastic modulus of $\mathrm{NF} /$ hydrogels. The mass erosion results in Fig. 2C also support this speculation, as they show that high NF content can inhibit disintegration of NF/hydrogels in an NFcontent dependent manner. Specifically, 1\%, 5\%, 10\%, 15\%, and $20 \%$ hydrogels showed average degradations of $57.14 \%$, $37.74 \%, 31.2 \%, 29.2 \%$, and $22.23 \%$ in comparison to the original mass, respectively, while the hydrogels without NF showed
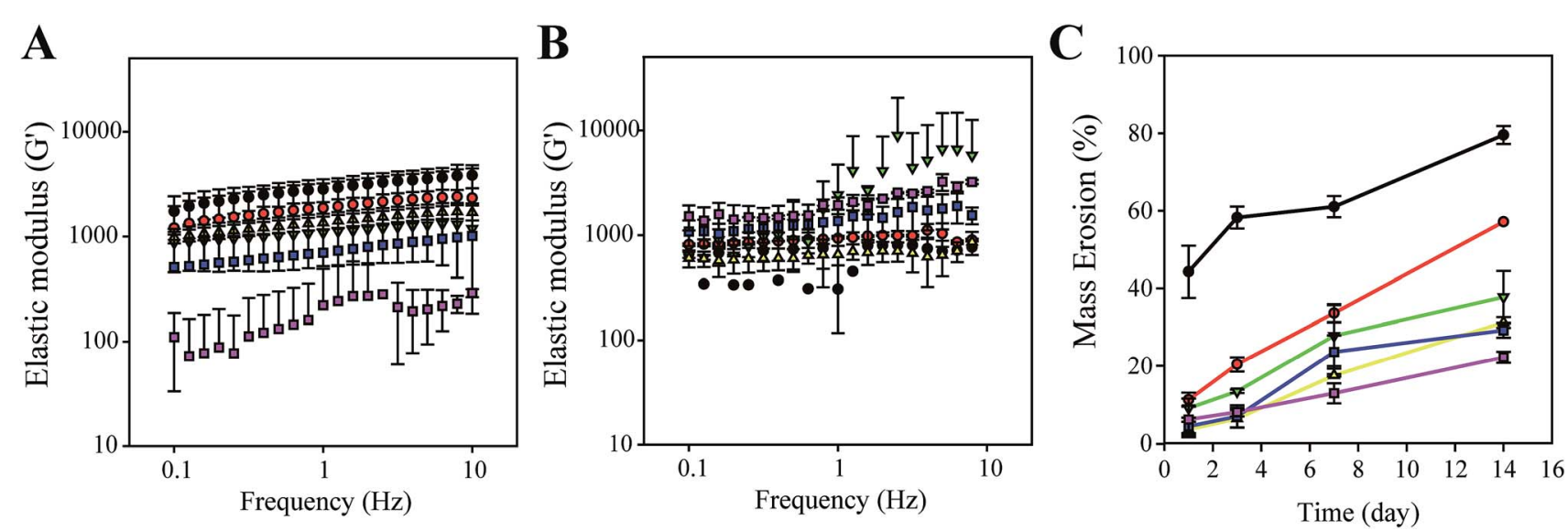

Fig. 2 Mechanical properties of NF/hydrogels incorporated with NIH3T3 for 7 days. Elastic modulus (G') of hydrogels at (A) day 0 and at (B) day 7.

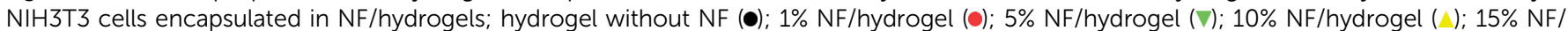
hydrogel $(\square) ; 20 \%$ NF/hydrogel $(\square)$ were incubated in DMEM with $10 \%$ FBS for 7 days and mounted on a rotating rheometer. (C) Mass erosions of NF/hydrogel encapsulating cells on 1, 3, 7 and 14 day. 
79.58\% degradation after 14 days. These results indicate that NF can contribute to the enhancement of mechanical properties of the matrix due to NF networks in NF/hydrogels mechanically holding the surrounding gelatin/alginate chains. Physicallycrosslinked hydrogels composed of alginate and gelatin show weaker physical properties than chemically-crosslinked hydrogels because non-covalent linkages such as electrostatic interactions and hydrogen bonding mediate the hydrogel formation in physical hydrogels. Furthermore, addition of any artifacts can interfere with the proper linkages among the polymer chains comprising hydrogels. ${ }^{20}$ Therefore, as shown in Fig. 2A, we also observed that the mechanical properties of the NF/ hydrogels are not higher than those without NF; however, due to the secreted ECM around NFs, NFs can tightly hold the hydrogel network and consequently attenuate mass erosion of overall structures. Additionally, water-swelling properties was gradually decreased when $\mathrm{NF}$ contents in $\mathrm{NF} /$ hydrogel increased (Fig. S2 $\dagger$ ).

To determine cell viability in NF/hydrogels, a MTT-based viability assay was performed on the cells cultivated in $\mathrm{NF} /$ hydrogels or hydrogels after 14 days as shown in Fig. 3. Cells in NF/hydrogels showed higher proliferation rates than those in hydrogels after 14 days and the degree of cell proliferation rate was dependent on the NF content in the NF/hydrogels, where those in $20 \% \mathrm{NF} /$ hydrogels showed the highest viabilities. Before 3 days of cultivation, the differences among NF/ hydrogels with various NF content levels were not significant; however, the viability of cells in $\mathrm{NF} /$ hydrogels was dramatically increased when the cultivation period reached the $14^{\text {th }}$ day. These high viabilities of cells in $\mathrm{NF} /$ hydrogels can be attributed both to low mass erosion as well as to the effects of the NF. During the cultivation period of 14 days, NF/hydrogels with higher NF content showed lower mass erosion rates than those with lower NF content. Therefore, due to the higher remaining volumes of the matrix, NF/hydrogels with higher NF content can act as more effective supportive matrices for cell proliferation than those with lower NF content.

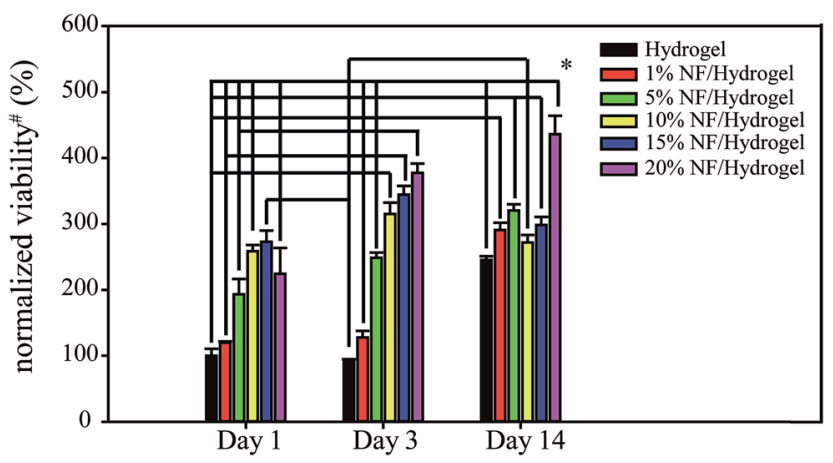

Fig. 3 Cell viability after 1, 3 and 14 days of cell cultivated in NF/ hydrogels. Each NF/hydrogels encapsulating NIH3T3 cells were homogenized at the day of the assay and subjected to a MTT-based viability. "The normalized viabilities are calculated by dividing the viability of respective sample by that in a hydrogel without NF at day 1 $(n=4)$. * indicates statistical significance level of $p<0.05$ by one way ANOVA.
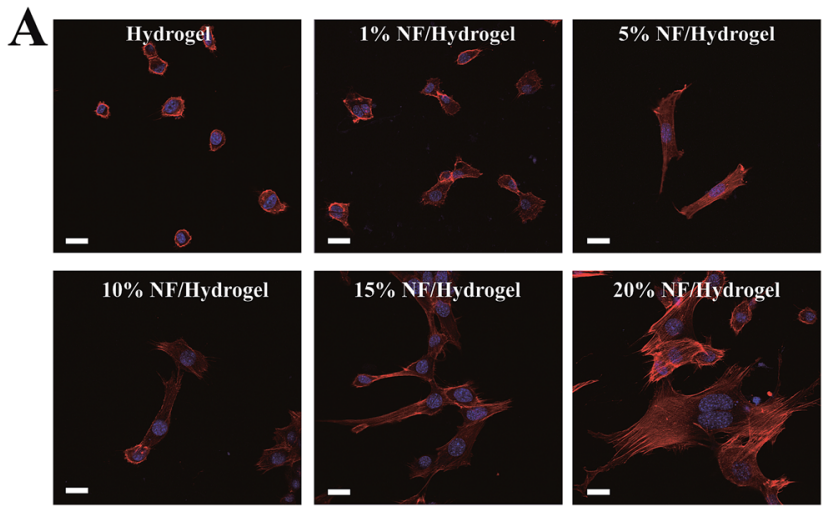

B

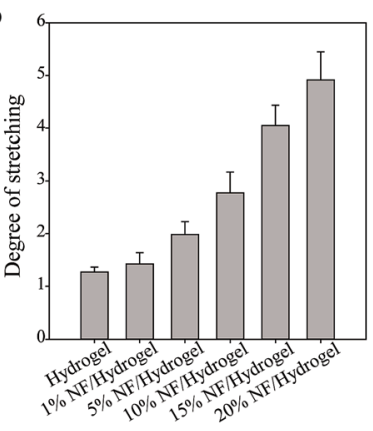

C

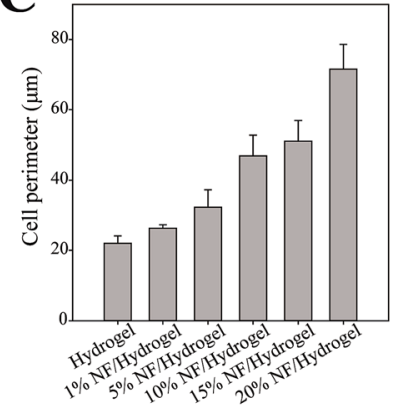

Fig. 4 Morphology of NIH3T3 cells in NF/hydrogels. (A) Confocal laser scanning microscopy of NIH3T3 cells in hydrogel, 1\% NF/hydrogel, $5 \%$ $\mathrm{NF} /$ hydrogel, $10 \% \mathrm{NF} /$ hydrogel, 15\% NF/hydrogel, and 20\% NF/ hydrogel were cultivated for $72 \mathrm{~h}$ and stained with DAPI (blue) and Alexa Fluor-Phalloidin (red) for visualizing nuclei and actin, respectively. (Scale bar $=20 \mu \mathrm{m}$.) (B) The degree of stretching $\left(D_{\mathrm{s}}\right)$ and $(C)$ the perimeters of each cell were calculated by image-analyzing the confocal images as described in Materials and Methods. More than 10 cells were counted and statistical analysis was performed.

We observed cell morphology in NF/hydrogels by CLSM as shown in Fig. 4A. When the nucleus and actin filaments were fluorescently labeled and visualized, cells showed significantly different morphologies according to the NF content in NF/ hydrogels; cells in hydrogels did not show the spreading or elongated shapes typical of fibroblastic cells. However, cells in $\mathrm{NF} /$ hydrogels were more likely to exhibit spreading and elongated morphology according to their NF content. Specifically, cells in $20 \% \mathrm{NF} /$ hydrogel showed the most elongated shapes and actin filaments were well defined as shown in Fig. 4A. Because the assembly of focal adhesions is associated with intracellular actin filaments, we speculate that cells cultivated in $20 \% \mathrm{NF} /$ hydrogel are most actively migrating and attaching on the surface..$^{21}$ Fahlgren et al., showed that human fibroblasts cultivated on biocompatible surfaces were stably captured and showed enhanced assembly of focal adhesions by elongation of F-actin. ${ }^{22}$ To quantify cell spreading and elongation, we analyzed the fluorescence images and quantified the degree of stretching and cell perimeters as shown in Fig. $4 \mathrm{~B}$ and C. ${ }^{22}$ The degree of cell stretching $\left(D_{\mathrm{S}}\right)$ and the cell perimeter increased in proportion to the NF content in NF/hydrogels; cells cultivated in $20 \% \mathrm{NF} /$ hydrogels showed 3-fold greater $D_{\mathrm{s}}$ and 3-fold longer cell perimeters than those in hydrogels. Because fibroblastic 
cells tend to show elongated shapes in favorable environments, many studies previously employed these parameters to assess the biocompatibility of specific surfaces toward cell attachment. NIH3T3 cells were usually used to investigate cell adhesion, spreading, and proliferation, because these cells have good expertise of adhesive properties such as integrin expression patterns, focal adhesion assembly, and spreading. ${ }^{\mathbf{2 3 , 2 4}}$ Thus, to explore cell-matrix interactions in NF/hydrogels, we herein employed NIH3T3 cells to easily confirm the feasibility of NF/ hydrogels as a 3-D scaffolds and confirmed that NF/hydrogels with higher NF content levels can provide more favorable environments for cell cultivation in 3-D environments than those without NF. It should be also noticed that visualizing 3-D cell morphology could not be obtained because of the higher scattering of NF in the hydrogels.

To determine the effects of NF content on cellular functions, we examine the collagen synthesis behaviors of NIH3T3 cells at the mRNA level (Fig. 5). In comparison to cells in hydrogels, those cultivated in NF/hydrogels showed higher expressions of collagen I and collagen II, which are the major collagen family proteins that fibroblastic cells secrete. Although we could not find significant differences among the various NF/hydrogels, semi-quantitative analysis indicated that cells in NF/hydrogels with higher NF content seemed to express higher amounts collagen I and collagen II mRNA; specifically, cells in hydrogels showed Col I/GAPDH and Col II/GAPDH ratios of 0.12 and 0.15 , respectively; cells in $10 \% \mathrm{NF} /$ hydrogels showed the highest

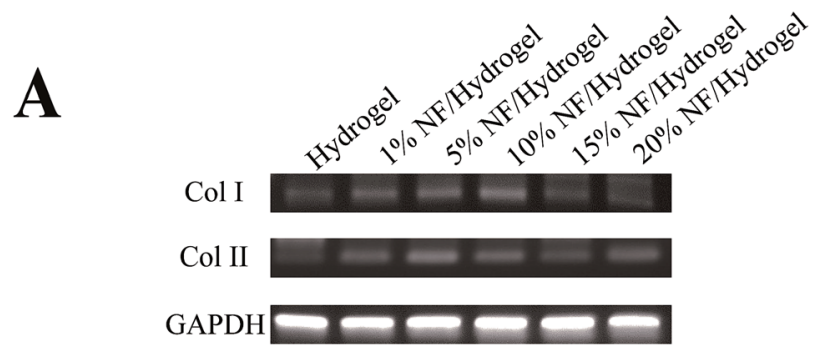

B

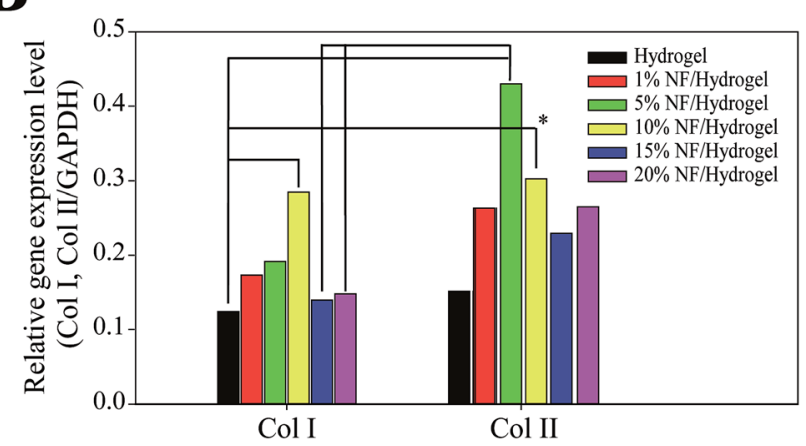

Fig. 5 RT-PCR of mRNA extracted from cells cultivated in NF/ hydrogels. Cells cultivated in NF/hydrogels at 14 days were harvested and subjected to (A) RT-PCR analysis of collagen I, and collagen II GAPDH of cells in hydrogel, $1 \% \mathrm{NF} /$ hydrogel, $5 \% \mathrm{NF} /$ hydrogel, $10 \% \mathrm{NF} /$ hydrogel, 15\% NF/hydrogel and 20\% NF/hydrogel. (B) Relative gene expression levels of NIH3T3 cells in hydrogels and NF/hydrogel were analyzed by image J. * indicates statistical significance level of $p<0.05$ by one way ANOVA. synthesis of Col I and Col II, where the ratios of 0.28 and 0.30 , respectively. Because nanofibrous structures have been regarded as a cell-friendly structure mimicking natural ECM, the fibrous structures in $\mathrm{NF} /$ hydrogels can allow cultivating cells to proliferate well in terms of increasing cell population as well as enhancing cellular functions such as secreting ECMs. However, it is interesting that cells in $10 \% \mathrm{NF} /$ hydrogel showed the higher expressions both of Col I and Col II than those in $15 \%$ or $20 \%$ $\mathrm{NF} /$ hydrogel. While proliferation and cell spreading were highest in cells in $20 \% \mathrm{NF} /$ hydrogel, it was expected that cell in $20 \% \mathrm{NF} /$ hydrogel showed the highest expression of collagen family. We speculate that higher proliferation observed in cells in $15 \%$ or $20 \% \mathrm{NF} /$ hydrogel can conversely attenuate protein synthesis of individual cell. Cells in 10\% NF/hydrogel are less densely packed and most population of cells can have cellmatrix contacts with $\mathrm{NF}$ in $\mathrm{NF} /$ hydrogel. However, relatively large population of cells in $15 \%$ or $20 \% \mathrm{NF} /$ hydrogel cannot be in a direct contact with NF because of the highly-proliferated cell populations and the effects of NF on cell functions can be weaker than those in $10 \% \mathrm{NF} /$ hydrogel. Therefore, to maximize the effect of NF on cell functions, sophisticated methods need to be designed to allow the cultivating cells to have maximum contacts with NF matrix throughout the cultivation period. Additionally, although we employed fibroblastic cell lines, similar studies indicate that these cells can be employed for determining the effects of biomaterials on cellular function. Type 1 collagen is synthesized in the osteoblast and fibroblast, and it has distinct effects on cell proliferation and strong fibrogenic ability on cells that are cultivated in the ECM. ${ }^{25,26}$ Thus, we can determine the potential effects of $\mathrm{NF}$ in $\mathrm{NF} /$ hydrogels by using NIH3T3 cells as a model cell line. The cellproduced ECM can serve as additional support to glue a mixture of NF and gelatin/alginate gel together. Previously studies also suggested the feasibility of fibrillar matrices as biomimetic scaffolds in 2-D cultivation. In osteoblasts cultivated on solid scaffolds and nanofibrous scaffolds, calcium concentrations of cells at nanofibrous scaffolds were higher than those at solid scaffolds after incubation. Fibroblasts cultivated on collagen-coated nanofibrous scaffolds expressed more of many kinds of proteins like keratin, filaggrin, and involucrin than cells grown on non-coated nanofibers. ${ }^{27,28}$ Thus, we anticipate that the current NF-fortified hydrogels can be candidate matrices for cell cultivation in 3-D fibrous structures mimicking natural ECM.

\section{Conclusions}

We fabricated nanofibril-fortified hydrogels with a mixture of hydrolyzed nanofibers and gelatin/alginate. Mechanical properties of NF/hydrogels were dependent on NF content in the matrix and cells cultivated in NF/hydrogels showed increased mechanical properties after 7 days. Cells cultivated on NF/ hydrogels exhibited more elongated and stretched morphology than those on hydrogels in an NF contentdependent manner. Gene expression levels of collagen I and II were notably higher in cells cultivated in NF/hydrogels. 


\section{Conflicts of interest}

There are no conflicts to declare.

\section{Acknowledgements}

This work supported by the National Research Foundation in Korea (NRF-2016H1D5A1908329).

\section{Notes and references}

1 D. Harjanto and M. H. Zaman, PLoS One, 2013, 8, e52509.

2 K. von der Mark, J. Park, S. Bauer and P. Schmuki, Cell Tissue Res., 2010, 339, 131.

3 S. Hinderer, S. L. Layland and K. Schenke-Layland, Adv. Drug Delivery Rev., 2016, 97, 260-269.

4 D. Jhala, H. Rather and R. Vasita, Biomater. Sci., 2016, 4, 1584-1595.

5 Y. I. Cho, J. S. Choi, S. Y. Jeong and H. S. Yoo, Acta Biomater., 2010, 6, 4725-4733.

6 S. Y. Chew, J. Wen, E. K. Yim and K. W. Leong, Biomacromolecules, 2005, 6, 2017-2024.

7 L. H. Nguyen, M. Gao, J. Lin, W. Wu, J. Wang and S. Y. Chew, Sci. Rep., 2017, 7, 42212.

8 M. Suhaeri, R. Subbiah, S. H. Kim, C. H. Kim, S. J. Oh, S. H. Kim and K. Park, ACS Appl. Mater. Interfaces, 2016, 9, 224-235.

9 J. Yang, C. Gong, F. K. Shi and X. M. Xie, J. Phys. Chem. B, 2012, 116, 12038-12047.

10 N. B. Shelke, P. Lee, M. Anderson, N. Mistry, R. K. Nagarale, X. M. Ma and S. G. Kumbar, Polym. Adv. Technol., 2016, 27, 42-51.

11 H. S. Kim and H. S. Yoo, Chem. Commun., 2015, 51, 306-309. 12 Y. H. Cho, Konyang Medical Journal, 2007, 7, 79-83.

13 Y. H. Huh, J. H. Ryu, S. Shin, D. U. Lee, S. Yang, K. S. Oh, J. K. Choi, W. K. Song and J. S. Chun, Gene, 2009, 448, 7-15.
14 K. Wang, K. C. Nune and R. D. K. Misra, Acta Biomater., 2016, 36, 143-151.

15 J. Solon, I. Levental, K. Sengupta, P. C. Georges and P. A. Janmey, Biophys. J., 2007, 93, 4453-4461.

16 W. H. Guo, M. T. Frey, N. A. Burnham and Y. L. Wang, Biophys. J., 2006, 90, 2213-2220.

17 A. J. Engler, S. Sen, H. L. Sweeney and D. E. Discher, Cell, 2006, 126, 677-689.

18 R. W. Tilghman, E. M. Blais, C. R. Cowan, N. E. Sherman, P. R. Grigera, E. D. Jeffery and J. T. Parsons, PLoS One, 2012, 7, e37231.

19 R. W. Tilghman, C. R. Cowan, J. D. Mih, Y. Koryakina, D. Gioeli, J. K. Slack-Davis and J. T. Parsons, PLoS One, 2010, 5, e12905.

20 J. Jang, J. Lee, Y. J. Seol, Y. H. Jeong and D. W. Cho, Composites, Part B, 2013, 45, 1216-1221.

21 M. L. Gardel, B. Sabass, L. Ji, G. Danuser, U. S. Schwarz and C. M. Waterman, J. Cell Biol., 2008, 183, 999-1005.

22 A. Fahlgren, C. Bratengeier, A. Gelmi, C. M. Semeins, J. Klein-Nulend, E. W. Jager and A. D. Bakker, PLoS One, 2015, 10, e0134023.

23 S. R. Ryoo, Y. K. Kim, M. H. Kim and D. H. Min, ACS Nano, 2010, 4, 6587-6598.

24 N. D. Gallant, J. R. Capadona, A. B. Frazier, D. M. Collard and A. J. García, Langmuir, 2002, 18, 5579-5584.

25 C. H. Liu, Y. Y. Hu, X. L. Wang, L. M. Xu and P. Liu, World J. Gastroenterol., 2000, 6, 361.

26 G. Karsenty and R. W. Park, Int. Rev. Immunol., 1995, 12, 177185.

27 K. M. Woo, J. H. Jun, V. J. Chen, J. Seo, J. H. Baek, H. M. Ryoo and P. X. Ma, Biomaterials, 2007, 28, 335-343.

28 V. J. Reddy, S. Radhakrishnan, R. Ravichandran, S. Mukherjee, R. Balamurugan, S. Sundarrajan and S. Ramakrishna, Wound Repair Regen., 2013, 21, 1-16. 